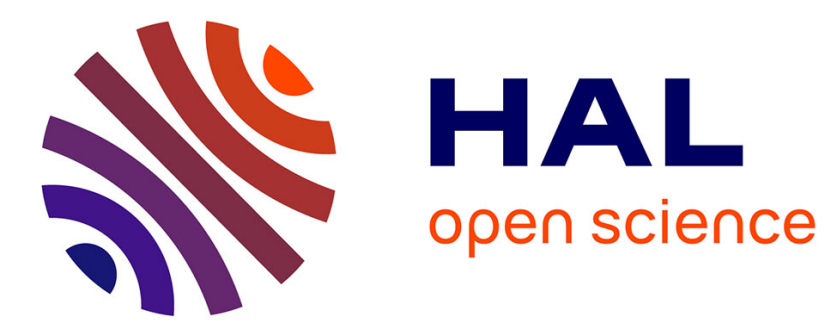

\title{
Gate Tunable Vertical Geometry Phototransistor based on Infrared HgTe Nanocrystals
}

\author{
Charlie Gréboval, Ulrich Nguétchuissi Noumbé, Audrey Chu, Yoann Prado,
} Adrien Khalili, Corentin Dabard, Tung Huu Dang, Silviu Colis, Julien Chaste, Abdelkarim Ouerghi, et al.

\section{To cite this version:}

Charlie Gréboval, Ulrich Nguétchuissi Noumbé, Audrey Chu, Yoann Prado, Adrien Khalili, et al.. Gate Tunable Vertical Geometry Phototransistor based on Infrared HgTe Nanocrystals. Applied Physics Letters, 2020, 117 (25), pp.251104. 10.1063/5.0032622 . hal-03086952

\section{HAL Id: hal-03086952 https://hal.science/hal-03086952}

Submitted on 23 Dec 2020

HAL is a multi-disciplinary open access archive for the deposit and dissemination of scientific research documents, whether they are published or not. The documents may come from teaching and research institutions in France or abroad, or from public or private research centers.
L'archive ouverte pluridisciplinaire HAL, est destinée au dépôt et à la diffusion de documents scientifiques de niveau recherche, publiés ou non, émanant des établissements d'enseignement et de recherche français ou étrangers, des laboratoires publics ou privés. 


\section{Gate Tunable Vertical Geometry Phototransistor based on Infrared HgTe Nanocrystals}

Charlie Gréboval ${ }^{1}$, Ulrich Nguétchuissi Noumbé², Audrey Chu' ${ }^{1}$, Yoann Prado ${ }^{1}$, Adrien Khalili ${ }^{1}$, Corentin Dabard ${ }^{1}$, Tung Huu Dang ${ }^{1}$, Silviu Colis ${ }^{2}$, Julien Chaste ${ }^{3}$, Abdelkarim Ouerghi ${ }^{3}$, JeanFrancois Dayen ${ }^{2,4}$ Emmanuel Lhuillier $^{1^{*}}$

'Sorbonne Université, CNRS, Institut des NanoSciences de Paris, INSP, F-75005 Paris, France ${ }^{2}$ Université de Strasbourg, CNRS, Institut de Physique et Chimie des Matériaux de Strasbourg (IPCMS), UMR 7504, 23 rue du Loess, Strasbourg, 67034, France.

${ }^{3}$ Centre de Nanosciences et de Nanotechnologies, CNRS, Université Paris-Saclay, C2N, Palaiseau 2110, France.

${ }^{4}$ Institut Universitaire de France, 1 rue Descartes, 75231 Paris cedex 05, France.

Abstract: Infrared nanocrystals are promising building blocks for the design of low-cost infrared sensors. Vertical geometry diode is, among possible geometries, the one that lead to the best performance so far. However, this geometry suffers from a lack of tunability after its fabrication, slowing down possible improvements. Here, we demonstrate gate control on a vertical diode in which the active layer is made of $\mathrm{HgTe} \mathrm{NCs}$ absorbing in the extended short-wave infrared $(2.5 \mu \mathrm{m})$. To reach this goal, we take advantage of the electrostatic transparency of graphene, combined with the high capacitance $\mathrm{LaF}_{3}$ ionic glass to design a gate tunable photodiode. The latter behaves as a work-function-tunable electrode which lets the gate-induced electric field tune the carrier density in the nanocrystal film. In particular, we show that the gate allows to tune the band profile leading to more efficient charge extraction and thus an enhanced-photoresponse ( $x 4$ compared to the device with floating gate). This work also demonstrates that photoelectron extraction can still be improved in the existing diode, by better controlling the doping profile of the diode.

Keywords: HgTe nanocrystal, infrared, photodiode, gate effect, mixed dimensionalities device.

*To whom correspondence should be sent: el@insp.upmc.fr 
HgTe nanocrystals (NCs) combining the semimetal nature of the bulk ${ }^{1,2}$ and quantum confinement ${ }^{3-}$ ${ }^{5}$, presents a unique spectral tunability in the infrared range. ${ }^{6}$ Beyond material absorption, $\mathrm{HgTe}$ NCs have been used for their IR light emitting properties ${ }^{7-12}$ as well as for their short ${ }^{13,14}$ and mid wave ${ }^{15,16}$ IR photoconduction properties. ${ }^{17}$ This has led to the demonstration of focal plane arrays operating in the short-18,19 and mid-wave infrared. ${ }^{20}$

Among possible device geometries, the vertical diode is the one that leads to the highest performance so far. The in-built electric field allows zero bias operation reducing the dark current as well as the noise. However, the number of structures that have been proposed to design a vertical geometry diode using $\mathrm{HgTe}$ NCs remains quite limited, ${ }^{21-24}$ and does not seem fully optimized. In particular, for an optical band gap in the 500 to $750 \mathrm{meV}$ range, the open circuit voltage is only a few tens of millivolts. This is far smaller than half of the band gap value commonly achieved for $\mathrm{PbS}$ quantum dots. Even though a part of this loss may directly be related to the traps in the material, ${ }^{25,26}$ it also suggests that the currently proposed diode stack remains sub-optimal.

The design of such diode is nevertheless challenging. Part of the open circuit voltage loss can directly come from the poor doping profile within the diode. Controlling the intrinsic doping in the NC film remains a difficult task and thus it will be of utmost interest to control the film carrier density using electrostatic gates. Field effect transistors based on $\mathrm{HgTe}$ films have been reported with various gate technologies such as dielectrics, ${ }^{27-29}$ electrolytes ${ }^{30}$ and even ionic glasses. ${ }^{31}$ However, all are based on planar geometry. Implementation of a gate in a vertical geometry appears far more challenging and remains unreported for $\mathrm{HgTe} \mathrm{NC}$ based diodes.

Our goal is to develop electrodes for the design of infrared NC-based diodes with the dual role of collecting carriers on one hand and controlling the carrier density within the film on the other hand. The development of such electrode faces a second challenge relating to its transparency. In most NC-based diodes, transparent conductive oxides (TCOs) are used. However, in the infrared TCOs present a strong absorption, requiring a decrease in their thicknesses at the cost of an increased contact resistance. Thus, alternative materials should be preferred. Here we explore graphene coupled to $\mathrm{LaF}_{3}$ ionic glass used as substrate and infrared transparent ${ }^{32}$ bottom electrode which can also be used to control the carrier density of the NC film. Compared to the works from Yao and coworkers, ${ }^{33,34}$ the switch from a conventional dielectric to the high capacitance gate allows us to expand the design of the doping landscape towards higher carrier density.

Coupling infrared $\mathrm{NCs}^{35,36}$ including $\mathrm{HgTe}^{37,38}$ to graphene and 2D materials ${ }^{39}$ is an approach that stimulated a growing activity over the past few years. So far, the most investigated geometry relies on a planar configuration ${ }^{13,40}$ where the graphene is used as a channel of a field effect transistor which is light sensitized by the NCs. ${ }^{41}$ The large mobility of graphene is used to generate gain and achieve very large responsivities (i.e. light to current conversion). However, the lack of band gap in graphene comes with a large dark conductance partly balancing the gain benefit. Here, we go further while exploring the use of the graphene large quantum capacitance. Contrary to metal or TCO electrodes, graphene does not screen the electric field induced by the $\mathrm{LaF}_{3}$ ionic glass. As a result, the gate below the 2D material can be used both to control the work function of the electrodes ${ }^{13,42,43}$ and to tune the carrier density within the NC film over its surface.

In this work, we demonstrate that exploiting the electrostatic transparency across mixeddimensional hetero-interfaces allows to use the back gate as a knob to control the current flow within the diode. The gate bias enables us to modify the band profile in the NC junction, that can now be switched from $n-p$ configuration to a $n^{+}-n-p$ configuration which is more favorable for charge extraction. This work confirms that $\mathrm{HgTe} \mathrm{NC}$-based diode performance can be further improved by optimizing the carrier density profile, in particular, on the $n$-side, as well as pinpoints this issue as a key element that future work will need to address. 
We start by growing HgTe NCs absorbing in the extended short wave infrared using Keuleyan's procedure. ${ }^{44}$ The particles (later called HgTe $\left.4 \mathrm{k}\right)$ present a clear excitonic feature at $4000 \mathrm{~cm}^{-1}(\approx 0.5$ $\mathrm{eV}$ or $2.5 \mu \mathrm{m}$ ), see Figure 1a. The nanocrystals have a planar tripodic shape according to transmission electron microscopy (see figure 1b). The nature of the majority carriers is also a central parameter for the following material integration and this can be probed using field effect transistor measurements. The latter is designed from electrodes onto a $\mathrm{LaF}_{3}$ substratre which is an ionic glass. ${ }^{45} \mathrm{LaF}_{3}$ provides a high capacitance ${ }^{31}\left(0.4 \mu \mathrm{F} . \mathrm{cm}^{-2}\right)$ while being all solid-state (i.e. without the constrains of electrolyte). Under such condition, the channel of $\mathrm{HgTe} \mathrm{NCs}$ shows an ambipolar behavior (Figure 1c) with both hole and electron conductions. The minimum of conductance occurs for a small negative gate bias ( $\mathrm{VGS}=-2 \mathrm{~V})$ meaning that there is a small inherent $n$ doping in this material. This picture is consistent with photoemission measurements on this material, which demonstrate that the Fermi level is close to the middle of the band gap and lies slightly closer to the conduction band. ${ }^{22}$

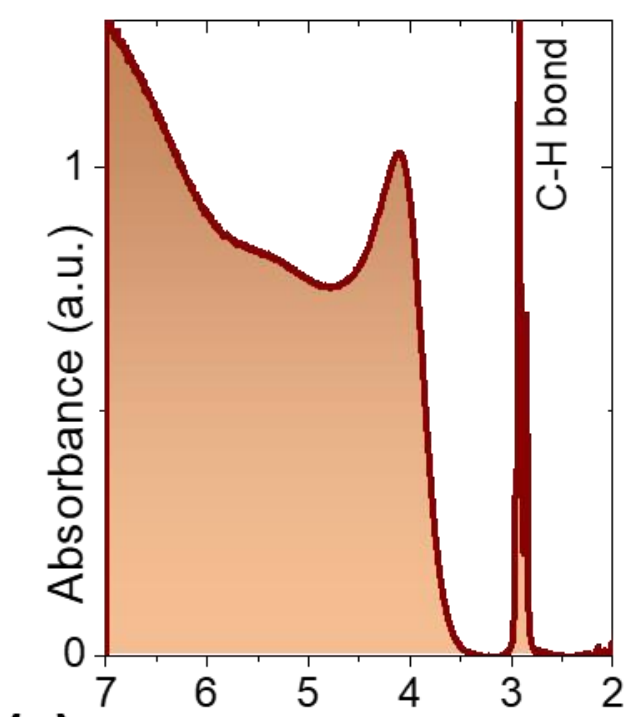

(a) Wavenumber $\left(10^{3} \times \mathrm{cm}^{-1}\right)$
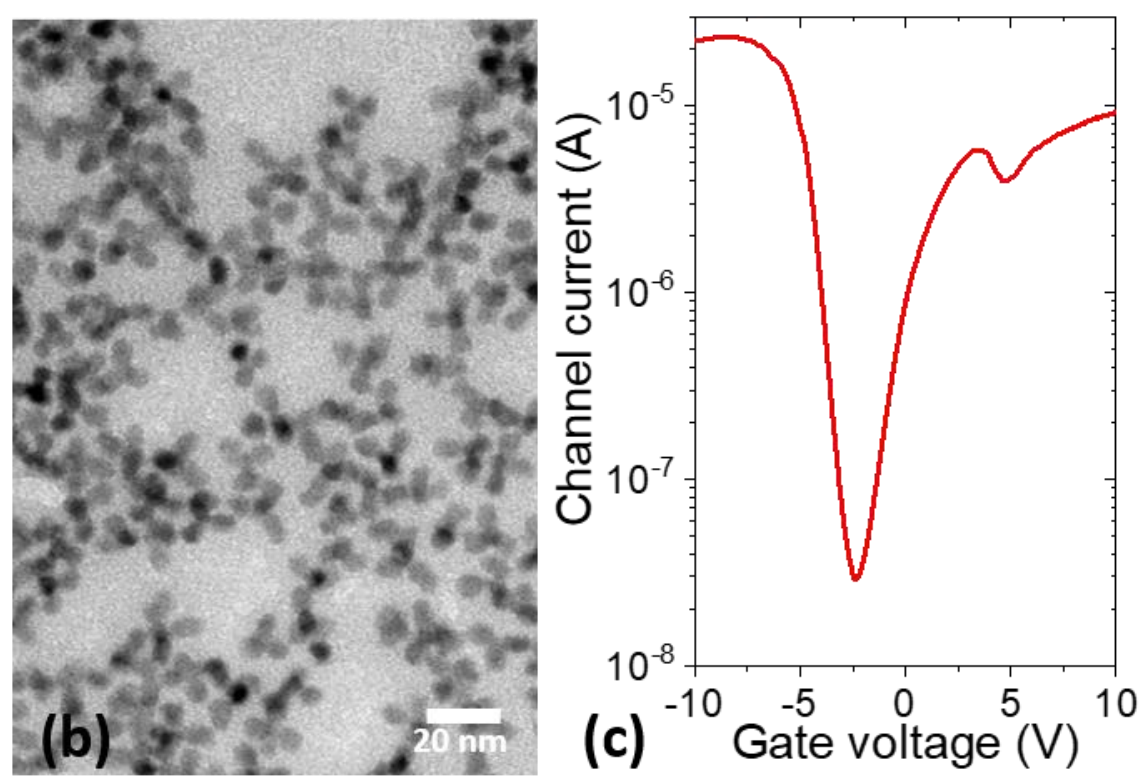

Figure $1 \mathrm{HgTe}$ infrared nanocrystals. a. Absorption spectrum of the HgTe NCs. b. Transmission electronic microscopy image of HgTe NCs associated with the spectrum of part a. c. Transfer curve (drain current as a function of the gate bias) for a thin film of HgTe NCs used as the channel of a field-effect transistor which gate is made of LaF $F_{3}$ ionic glass.

The bottom electrode of the diode made of graphene was also characterized independently (ie without the capping HgTe NC layer). The graphene is a CVD-grown graphene which has been transferred onto the $\mathrm{LaF}_{3}$ substrate. As expected for graphene, we also observe an ambipolar behavior (Figure 2a) for this material under gate bias application, with a minimum of conductance corresponding to the Dirac point observed for $\mathrm{V}_{\mathrm{GS}}=-4 \mathrm{~V}$. The graphene is then patterned as stripes, all connected to a gold contact, see Figure $2 \mathrm{~b}$. Using Raman mapping of the $2 \mathrm{D}$ mode of graphene (Figure 2C), we check that stripes are well defined. The processing of graphene electrode under stripes allows simultaneous charge collection, while leaving part of the film in direct contact with the gate to further enhance the carrier density tunability within the NC film. 


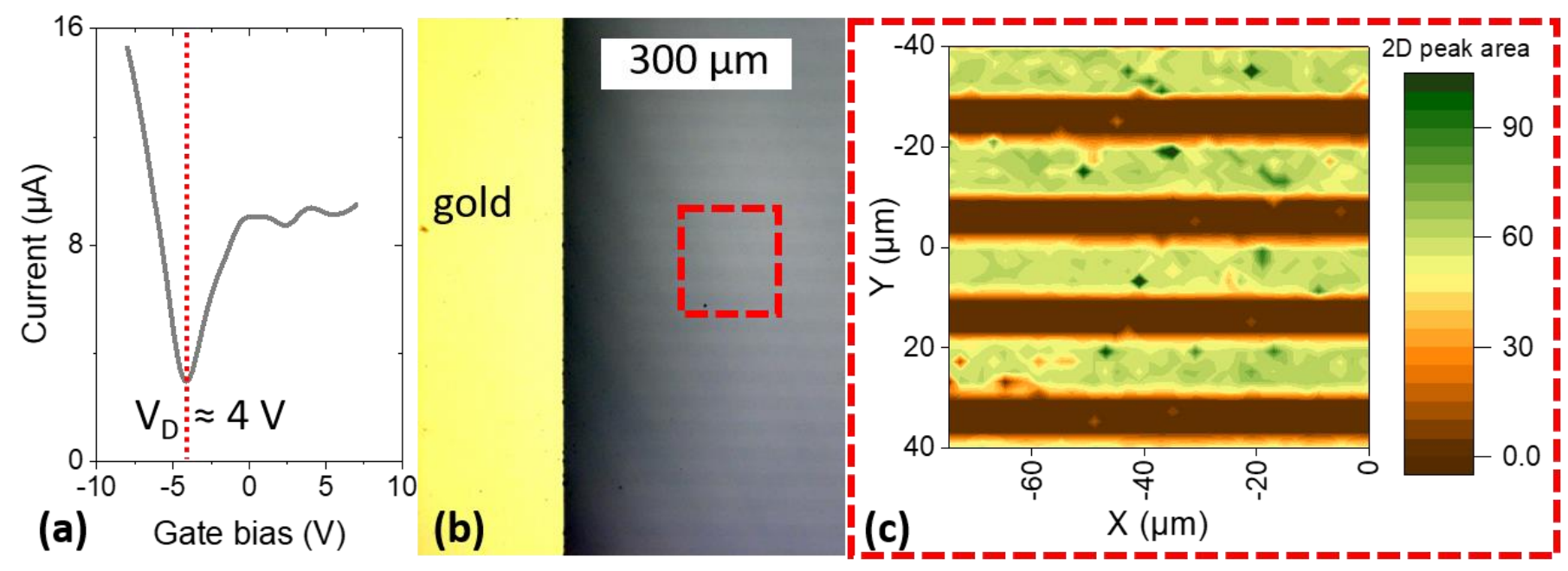

Figure 2 Graphene as infrared transparent, work function tunable electrode. a. Transfer curve (drain current as a function of the gate bias) for a graphene layer used as a channel of a field-effect transistor-which gate is made of $\mathrm{LaF}_{3}$ ionic glass. b. Image of the patterned graphene deposited onto a LaF 3 substrate and connected to a gold contact. $c$. Map of the intensity of the graphene in $2 D$ Raman mode of graphene for the electrode deposited on LaF. On this map the substrate is brown while the graphene is yellow green

We then design the diode according to the scheme of Figure 3a. A thin $(35 \mathrm{~nm})$ ITO layer is deposited on one side of a double side polished $\mathrm{LaF}_{3}$ substrate. The latter will be used as IR transparent gate electrode. The $\mathrm{LaF}_{3}$ substrate provides two functionalities: an IR transparent bottom substrate and a high capacitance gate medium. In $\mathrm{LaF}_{3}$, the gating results from $\mathrm{F}^{-}$vacancies displacement, optimal operating condition is in the $200-250 \mathrm{~K}$ range $(220 \mathrm{~K}$ is used as operating in the following of the paper). Above $250 \mathrm{~K}$ the leakage in the gate are high and compete with the channel flow, while below $180 \mathrm{~K}$, the vacancies freeze and the gate tunability is lost. On the other side of the substrate, the CVD-grown graphene is transferred and patterned as stripes, see figure $2 \mathrm{~b}$ and $\mathrm{c}$. The absorbing layer is made of a $300 \mathrm{~nm}$ film of $\mathrm{HgTe} 4 \mathrm{k}$ (i.e. presenting a band gap at $2.5 \mu \mathrm{m}$ ). On top of the $\mathrm{HgTe}$ $4 \mathrm{k}$ film, a thin layer of $\mathrm{HgTe} 7 \mathrm{k}$ (i.e. with a band-edge at $7000 \mathrm{~cm}^{-1}$ or $0.87 \mathrm{eV}$ ) is deposited. Finally, a top gold contact is used as a hole extractor.

In $\mathrm{HgTe}$, the strong asymmetry of effective mass between the valence and the conduction bands makes that most of the quantum confinement occurs in the conduction band. As the result of the heavy mass of the hole, the valence band is poorly affected by the confinement (i.e. the valence band weakly shifted when confinement is introduced) and it makes that various sizes of $\mathrm{HgTe} \mathrm{NCs}$ present quasi resonant valence band. ${ }^{22}$ The layer of $\mathrm{HgTe} 7 \mathrm{k}$ NCs with a larger band gap $\left(7000 \mathrm{~cm}^{-}\right.$ ${ }^{1}$ or $0.87 \mathrm{eV}$ ) on top of $\mathrm{HgTe} 4 \mathrm{k}$ is used as a unipolar barrier ${ }^{22}$ preventing electron diffusion towards the top electrode, while leaving the photo-holes flow.

To confirm the effect of the gate, we measure the current flow through the diode while the gate bias is tuned (the equivalent of a transfer curve in a field-effect transistor), see Figure 3b. Under a gate bias application, we observe a clear modulation of the current, typically by one order of magnitude. This confirms that the gate electrode can be used to tune the carrier density in the vicinity of the graphene electrode. 


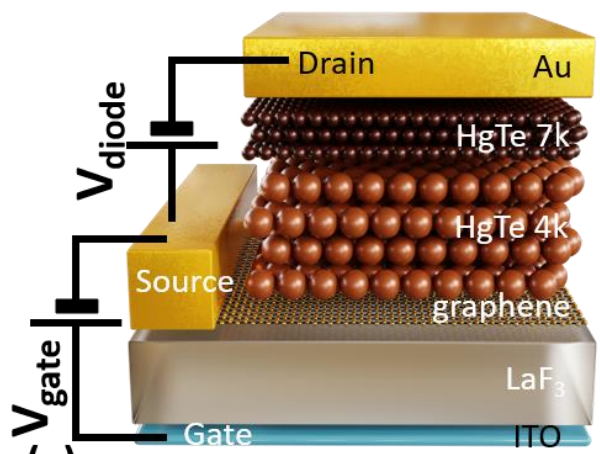

(a)
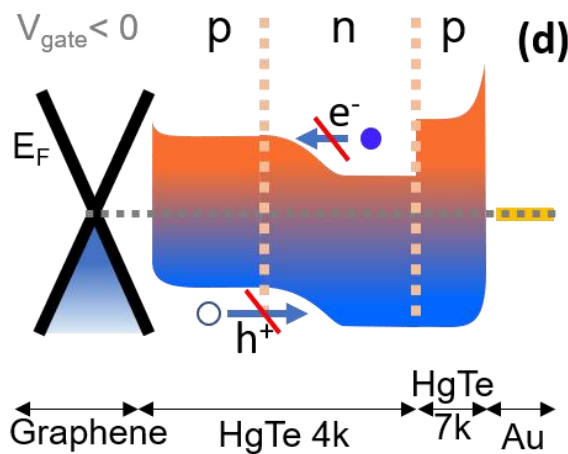

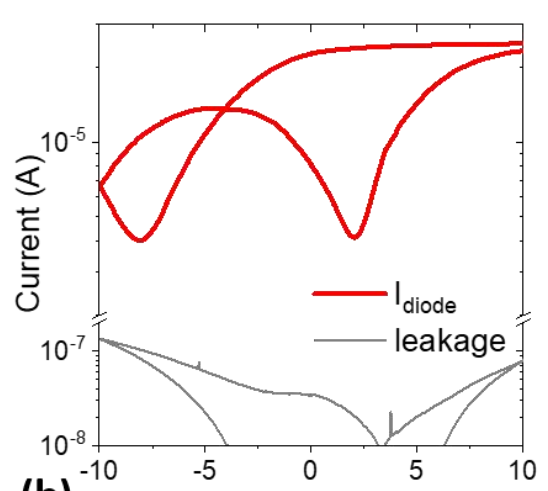

(b)

Gate voltage (V)

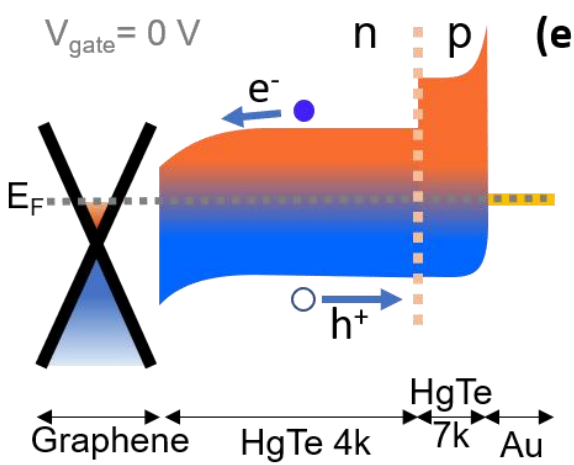

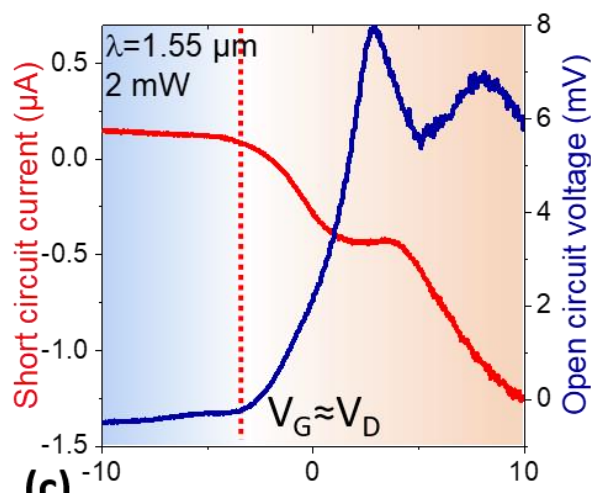

Gate voltage (V)

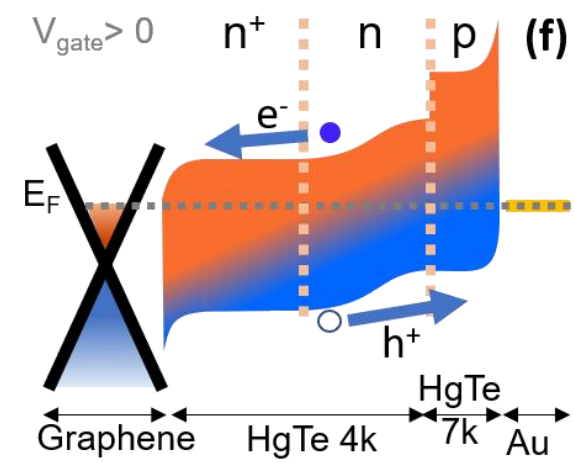

Figure 3 Gate tunable vertical geometry infrared diode. a. Sketch of the gate tunable diode based on the ITO/LaF3/graphene/HgTe $4 \mathrm{k} / \mathrm{HgTe} \mathrm{k} / \mathrm{gold}$ stack. $b$. Current through the diode while the gate bias is tuned $\left(V_{\text {diode }}=0.1\right.$ $V)$. c. Short circuit current and open circuit voltage for the diode under illumination $(\lambda=1.55 \mu \mathrm{m}-P=2 \mathrm{~mW}$ ) as a function of gate bias. Band structure of the diode under negative gate bias (part d.), $0 \mathrm{~V}$ bias (part e.) and positive gate bias (part f.). Measurements are conducted at $220 \mathrm{~K}$.

The band profile associated with this diode structure at $0 \mathrm{~V}$ is given in Figure $3 \mathrm{e}$. The slightly smaller work function of graphene (4.4-4.5eV) compared to $\mathrm{HgTe} 4 \mathrm{k}^{19,22}(4.6-4.7 \mathrm{eV})$ induces a downward band bending for $\mathrm{HgTe} 4 \mathrm{k}$ at its interface with graphene, while the trend is opposite at the $\mathrm{HgTe} 7 \mathrm{k} / \mathrm{gold}$ interface. Under positive gate bias application, the $n$-type nature of the $\mathrm{HgTe} 4 \mathrm{k} \mathrm{NC}$ thin film is reinforced creating a $n^{+} n p$ band profile which is expected to ease hole and electron extraction, see Figure 3f. On the other hand, when a negative gate bias is applied, holes are injected and a pnp band profile is generated, see Figure $3 \mathrm{~d}$. In this case a very poor photo-charge extraction is anticipated.

These predictions are experimentally confirmed by measuring the photocurrent spectrum, see figure 4a. We clearly observe that the gate bias drives the magnitude of the photocurrent while the bias over the diode is kept constant. No photoresponse is observed while the diode is operated in the pnp configuration. We also clearly observe an enhancement by a factor $\approx 4$ of the photocurrent magnitude as positive gate bias is applied.

The gate effect is quantitatively better monitored by measuring the short circuit current (Isc) and the open circuit voltage $\left(V_{O C}\right)$, see Figure $3 c$ and IV curves in Figure $S 5$. The pnp regime $\left(V_{G}<0\right)$ is characterized by very low performance: $V_{0 c}<1 \mathrm{mV}$ and weak photocurrent corresponding to responsivity of the order of $50 \mu \mathrm{A} . \mathrm{W}^{-1} @ 1.55 \mu \mathrm{m}$, which is weak compared to best diodes based on graphene/HgTe NCs. ${ }^{32}$ As the gate bias is tuned, we observe a change of sign for the Voc and Isc. This confirms the change of nature for the majority carriers from holes to electrons as gate bias is increased. The gate bias where the open circuit change of sign is $-4 \mathrm{~V}$ matching well with the Dirac point of graphene. For higher gate biases ( $\mathrm{n}^{+} \mathrm{np}$ regime), the open circuit voltage reaches $6 \mathrm{mV}$,

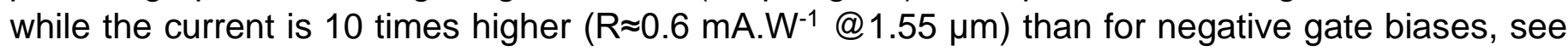


Figure 3c. Time response of the diode has been measured and found to be fast $(<100 \mathrm{~ms}$, setup limited), see Figure 4b.

Our results demonstrate that increasing the doping level of the NC absorbing layer enables an improvement of the diode performance. It also confirms that in current NC diodes the electron extraction is not as favorable as it could be if more strongly $n$-doped HgTe NCs might be synthetized while keeping the band gap constant.
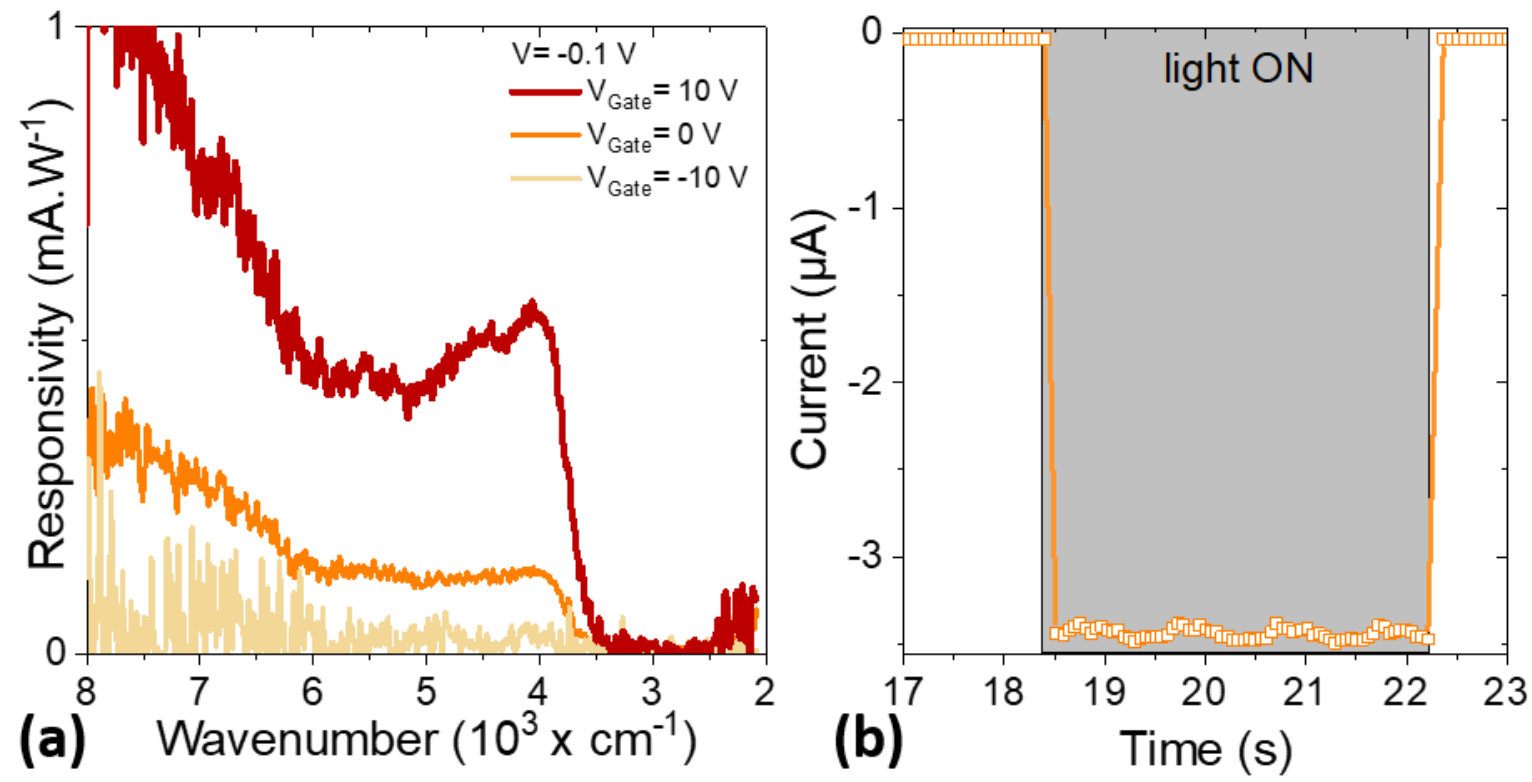

Figure 4 Infrared detection using the gate tunable diode. a. Spectral response of the gate tunable diode operated under three gate biases, while the diode bias is set to $-0.1 \mathrm{~V}$. Curve have been normalized to take into account the amplifier gain. $b$. Photocurrent under $0 \mathrm{~V}$ diode bias and $10 \mathrm{~V}$ gate bias while is the illumination $(\lambda=1.55 \mu \mathrm{m}-P=2 \mathrm{~mW})$ is turned on and off. Measurements are conducted at $220 \mathrm{~K}$

To summarize, gate control is commonly integrated in planar geometries for field effect transistors; but the use of gates in HgTe NC-based photodiodes remains unexplored. Here, we demonstrate the fabrication of a gate tunable photodiode architecture based on $\mathrm{HgTe} \mathrm{NCs}$ and a mixed-dimensional vertical stack. The combination of high capacitance $\mathrm{LaF}_{3}$ ionic glass and graphene is used as an infrared transparent electrode that allows us to adjust electrostatically the band profile of the NCs absorbing layer after fabrication. We demonstrate that in the floating condition (i.e. without gate), the band profile does not lead to the optimal condition for charge extraction. This work suggests that additional research will be needed to design better $n$-doped $\mathrm{HgTe}$ NCs to obtain optimized photoelectron extraction. Moreover, as demonstrated here on a first device generation, 2D-0D vertical heterostructures reveal a promising system to introduce electrostatic engineering of the diode absorbing layer.

See the supplementary material for information about nanocrystal growth, material characterization, device fabrication and device characterization.

The project is supported by ERC starting grant blackQD (grant $n^{\circ} 756225$ ). We acknowledge the use of clean-room facilities from the "Centrale de Proximité Paris-Centre" and from STnano platform. This work has been supported by the Region lle-de-France in the framework of DIM Nano-K (grant dopQD). This work was supported by French state funds managed by the ANR within the 
Investissements d'Avenir programme by Labex Matisse (ANR-11-IDEX-0004-02) and Labex NIE (ANR-11-LABX-0058, and ANR-10-IDEX-0002-02). ANR also fund grants FRONTAL (ANR-19CE09-0017), IPER-Nano2 (ANR-18CE30-0023-01), Copin (ANR-19-CE24-0022), Graskop (ANR19-CE09-0026), NITQuantum. AC thanks Agence Innovation Defense for PhD funding.

The authors declare no competing interests.

The data that supports the findings of this study are available within the article and its supplementary material.

\section{References}

${ }^{1}$ A. Delin and T. Klüner, Phys. Rev. B 66, 035117 (2002).

${ }^{2}$ A. Svane, N.E. Christensen, M. Cardona, A.N. Chantis, M. van Schilfgaarde, and T. Kotani, Phys. Rev. B 84, 205205 (2011).

${ }^{3}$ E. Lhuillier, S. Keuleyan, and P. Guyot-Sionnest, Nanotechnology 23, 175705 (2012).

${ }^{4}$ N. Moghaddam, C. Gréboval, J. Qu, A. Chu, C. Livache, S.-S. Chee, A. Khalili, X.Z. Xu, B. Baptiste, S. Klotz, G. Fishman, F. Capitani, S. Ithurria, S. Sauvage, and E. Lhuillier, J. Phys Chem C 123, 24360 (2020).

${ }^{5}$ G. Allan and C. Delerue, Phys. Rev. B - Condens. Matter Mater. Phys. 86, 165437 (2012).

${ }^{6}$ N. Goubet, A. Jagtap, C. Livache, B. Martinez, H. Portalès, X.Z. Xu, R.P.S.M. Lobo, B. Dubertret, and E. Lhuillier, J. Am. Chem. Soc. 140, 5033 (2018).

${ }^{7}$ A. Rogach, S.V. Kershaw, M. Burt, M.T. Harrison, A. Kornowski, A. Eychmüller, and H. Weller, Adv. Mater. 11, 552 (1999).

${ }^{8}$ A.A. Sergeev, D.V. Pavlov, A.A. Kuchmizhak, M.V. Lapine, W.K. Yiu, Y. Dong, N. Ke, S. Juodkazis, N. Zhao, S.V. Kershaw, and A.L. Rogach, Light Sci. Appl. 9, 16 (2020).

${ }^{9}$ P. Geiregat, A.J. Houtepen, L.K. Sagar, I. Infante, F. Zapata, V. Grigel, G. Allan, C. Delerue, D. Van

Thourhout, and Z. Hens, Nat. Mater. 17, 35 (2018).

${ }^{10}$ D.S. Koktysh, N. Gaponik, M. Reufer, J. Crewett, U. Scherf, A. Eychmüller, J.M. Lupton, A.L. Rogach, and J. Feldmann, ChemPhysChem 5, 1435 (2004).

11 J. Qu, P. Rastogi, C. Gréboval, D. Lagarde, A. Chu, C. Dabard, A. Khalili, H. Cruguel, C. Robert, X.Z. Xu, S. Ithurria, M.G. Silly, S. Ferré, X. Marie, and E. Lhuillier, Nano Lett. 20, 6185 (2020).

12 É. O'Connor, A. O'Riordan, H. Doyle, S. Moynihan, A. Cuddihy, and G. Redmond, Appl. Phys. Lett. 86, 201114 (2005).

13 U.N. Noumbé, C. Gréboval, C. Livache, A. Chu, H. Majjad, L.E. Parra López, L.D.N. Mouafo, B. Doudin, S. Berciaud, J. Chaste, A. Ouerghi, E. Lhuillier, and J.-F. Dayen, ACS Nano 14, 4567 (2020).

${ }^{14}$ Y. Dong, M. Chen, W.K. Yiu, Q. Zhu, G. Zhou, S.V. Kershaw, N. Ke, C.P. Wong, A.L. Rogach, and N. Zhao, Adv. Sci. 7, 2000068 (2020).

${ }^{15}$ M.E. Cryer and J.E. Halpert, ACS Photonics 5, 3009 (2018).

${ }^{16}$ S. Keuleyan, E. Lhuillier, V. Brajuskovic, and P. Guyot-Sionnest, Nat. Photonics 5, 489 (2011).

${ }^{17}$ E. Lhuillier and P. Guyot-Sionnest, IEEE J. Sel. Top. Quantum Electron. 23, 6000208 (2017).

${ }^{18}$ C. Gréboval, S. Ferre, V. Noguier, A. Chu, J. Qu, S.-S. Chee, G. Vincent, and E. Lhuillier, ArXiv200111554 Cond-Mat Physicsphysics (2020). https://arxiv.org/abs/2001.11554

${ }^{19}$ A. Chu, B. Martinez, S. Ferré, V. Noguier, C. Gréboval, C. Livache, J. Qu, Y. Prado, N. Casaretto, N. Goubet, H. Cruguel, L. Dudy, M.G. Silly, G. Vincent, and E. Lhuillier, ACS Appl. Mater. Interfaces 11, 33116 (2019).

${ }^{20}$ C. Buurma, R.E. Pimpinella, A.J. Ciani, J.S. Feldman, C.H. Grein, and P. Guyot-Sionnest, in Opt. Sens. Imaging Photon Count. Nanostructured Devices Appl. 993303 (2016).

${ }^{21}$ M.M. Ackerman, X. Tang, and P. Guyot-Sionnest, ACS Nano 12, 7264 (2018).

22 A.M. Jagtap, B. Martinez, N. Goubet, A. Chu, C. Livache, C. Gréboval, J. Ramade, D. Amelot, P. Trousset,

A. Triboulin, S. Ithurria, M.G. Silly, B. Dubertret, E. Lhuillier, ACS Photonics 5, 4569 (2018). 
${ }^{23}$ B. Martinez, J. Ramade, C. Livache, N. Goubet, A. Chu, C. Gréboval, J. Qu, W.L. Watkins, L. Becerra, E. Dandeu, J.L. Fave, C. Méthivier, E. Lacaze, and E. Lhuillier, Adv. Opt. Mater. 7, 1900348 (2019).

${ }^{24}$ M.M. Ackerman, M. Chen, and P. Guyot-Sionnest, Appl. Phys. Lett. 116, 083502 (2020).

${ }^{25}$ C.-H.M. Chuang, A. Maurano, R.E. Brandt, G.W. Hwang, J. Jean, T. Buonassisi, V. Bulović, and M.G. Bawendi, Nano Lett. 15, 3286 (2015).

${ }^{26}$ S. Pradhan, M. Dalmases, and G. Konstantatos, J. Phys. Chem. Lett. 10, 3029 (2019).

${ }^{27}$ E. Lhuillier, S. Keuleyan, P. Zolotavin, and P. Guyot-Sionnest, Adv. Mater. 25, 137 (2013).

${ }^{28}$ X. Lan, M. Chen, M.H. Hudson, V. Kamysbayev, Y. Wang, P. Guyot-Sionnest, and D.V. Talapin, Nat. Mater. 19, 323 (2020).

29 J. Jang, K. Cho, S.H. Lee, and S. Kim, Nanotechnology 19, 015204 (2007).

${ }^{30}$ C. Livache, E. Izquierdo, B. Martinez, M. Dufour, D. Pierucci, S. Keuleyan, H. Cruguel, L. Becerra, J.L. Fave, H. Aubin, A. Ouerghi, E. Lacaze, M.G. Silly, B. Dubertret, S. Ithurria, and E. Lhuillier, Nano Lett. 17, 4067 (2017).

${ }^{31}$ C. Gréboval, U. Noumbe, N. Goubet, C. Livache, J. Ramade, J. Qu, A. Chu, B. Martinez, Y. Prado, S. Ithurria, A. Ouerghi, H. Aubin, J.-F. Dayen, and E. Lhuillier, Nano Lett. 19, 3981 (2019).

${ }^{32}$ X. Tang, M. Chen, A. Kamath, M.M. Ackerman, and P. Guyot-Sionnest, ACS Photonics 7, 1117 (2020).

${ }^{33}$ Y. Che, Y. Zhang, X. Cao, X. Song, H. Zhang, M. Cao, H. Dai, J. Yang, G. Zhang, and J. Yao, Appl. Phys. Lett. 109, 263101 (2016).

${ }^{34}$ X. Song, Y. Zhang, H. Zhang, Y. Yu, M. Cao, Y. Che, H. Dai, J. Yang, X. Ding, and J. Yao, Nanotechnology 28, 145201 (2017).

${ }^{35}$ G. Konstantatos, M. Badioli, L. Gaudreau, J. Osmond, M. Bernechea, F.P.G. de Arquer, F. Gatti, and F.H.L. Koppens, Nat. Nanotechnol. 7, 363 (2012).

${ }^{36}$ Y. Zhang, X. Song, R. Wang, M. Cao, H. Wang, Y. Che, X. Ding, and J. Yao, Nanotechnology 26, 335201 (2015).

37 M. Grotevent, C.U. Hail, S. Yakunin, D. Bachmann, M. Calame, D. Poulikakos, M.V. Kovalenko, and I. Shorubalko, (2020). https://doi.org/10.26434/chemrxiv.12871331.v1

${ }^{38}$ X. Tang and K.W.C. Lai, ACS Appl. Nano Mater. 2, 6701 (2019).

${ }^{39}$ N. Huo, S. Gupta, and G. Konstantatos, Adv. Mater. 29, 1606576 (2017).

${ }^{40}$ I. Nikitskiy, S. Goossens, D. Kufer, T. Lasanta, G. Navickaite, F.H.L. Koppens, and G. Konstantatos, Nat. Commun. 7, 11954 (2016).

${ }^{41}$ D. Spirito, S. Kudera, V. Miseikis, C. Giansante, C. Coletti, and R. Krahne, J. Phys. Chem. C 119, 23859 (2015).

42 Y. Che, Y. Zhang, X. Cao, H. Zhang, X. Song, M. Cao, Y. Yu, H. Dai, J. Yang, G. Zhang, and J. Yao, ACS Appl. Mater. Interfaces 9, 32001 (2017).

43 U.N. Noumbé, C. Gréboval, C. Livache, T. Brule, B. Doudin, A. Ouerghi, E. Lhuillier, and J.-F. Dayen, Adv. Funct. Mater. 29, 1907462 (2019).

${ }^{44}$ S. Keuleyan, E. Lhuillier, and P. Guyot-Sionnest, J. Am. Chem. Soc. 133, 16422 (2011).

${ }^{45}$ C.L. Wu, H. Yuan, Y. Li, Y. Gong, H.Y. Hwang, and Y. Cui, Nano Lett. 18, 2387 (2018). 